\title{
The Design and Implementation of High Precision and Low Power Wireless Temperature Sensor
}

\author{
Yanping Wang ${ }^{1}$, Hanxi $\mathrm{Li}^{1}$, Yuchen Wang ${ }^{2}$ and Huan $\mathrm{Le}^{1}$ \\ ${ }^{1}$ China Tobacco Zhejiang Industrial Co., LTD \\ ${ }^{2}$ Hangzhou Xuejun High School
}

\begin{abstract}
This paper presents a design method of high precision wireless temperature sensor, the sensor consists of 16 high-performance low-power processor MSP430F149, Sub-1G RF transceiver chip SI4432, four-wire positive temperature coefficient temperature sensitive resistor PT100, the high resolution of AD conversion chip ADS1246 and other core components, temperature range $-40{ }^{\circ} \mathrm{C} \sim 80{ }^{\circ} \mathrm{C}$, resolution $0.01{ }^{\circ} \mathrm{C}$. The sensor can be collected if the temperature information stored locally or transmitted in real time to the control terminal, effectively overcome the wired sensor in the measurement limitations. In view of the technical characteristics of Sub-1G, the design method of high precision wireless temperature sensor in performance and power consumption is discussed.
\end{abstract}

\section{Keywords-low power high precision}

\section{INTRODUCTION}

Tobacco fermentation and mellowing process are very important factors for the tobacco quality. The inner temperature of the compressed tobacco package imbalance often heralds the tobacco may be bad. So, detecting tobacco package's inner temperature changing can be indirectly measured the tobacco package's corresponding state. When the temperature of the tobacco package is higher than the environments a certain value (normal $3^{\circ}-5^{\circ}$, or higher), the tobacco package may have taken place in mildew or carbonization, and it has affected the quality of tobacco leaf. Normally, tobacco mellowing process will continue 18 months to 36 months. It is very important to monitoring the inner temperature of the tobacco package by using high precision temperature sensor. Cabling temperature checking system is complex and not easy to maintain in tobacco warehouse, and there has a new solution by remote wireless transmission system. Sub-1GHZ communication, as a kind of popular narrowband communication technology, gradually popularized in the field of sensor. Sub - 1 GHZ technology combining with distributed signal acquisition, processing and with high resolution $\mathrm{AD}$ sampling technology to design the wireless temperature sensor with high precision, it can be convenient, real-time transmitted to the temperature of the object being measured in a reliable way to control terminal. To establish wireless sensor network (WSN) by private wireless communication protocol stack able to adapt to more complex measurement field.

\section{SYSTEM DESIGN}

\section{A. Hardware Subsystem Design}

Hardware subsystem mainly includes two major part , temperature sensor and intelligent terminal router. Temperature sensor terminal including: radio frequency communication, a temperature measurement, the MCU control unit. The system structure just like Figure I:

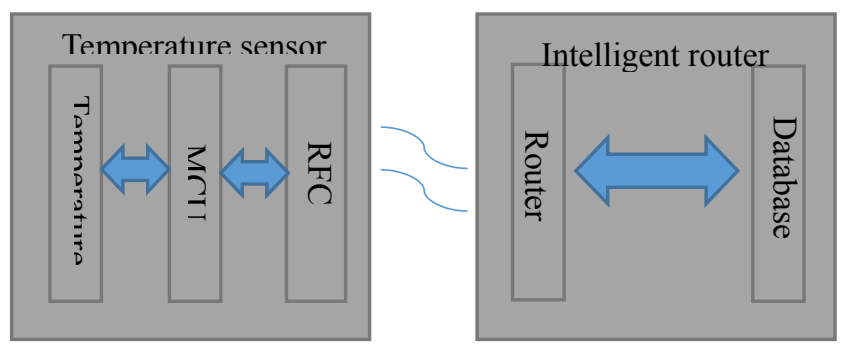

FIGURE I. THE SYSTEM STRUCTURE

1) Radio frequency communication: The existing wireless communication technology such as WIFI and bluetooth, usually did not fit the requirements of low power design, and ZigBee communication technology has high cost, hardware baseband spectrum limit shortcoming. ZigBee also has low wireless signal diffraction properties, where the tobacco warehouse always is filled in tobacco packages. So in this design we choose Silicon-Labs' chip which working in ISM frequency band, the SI4432 as the RF transceiver chip based on the Sub - $1 \mathrm{GHZ}$ technology, and using private wireless communication protocol networking, frequency set to 433 MHZ. The Communication circuit is just like Figure II:

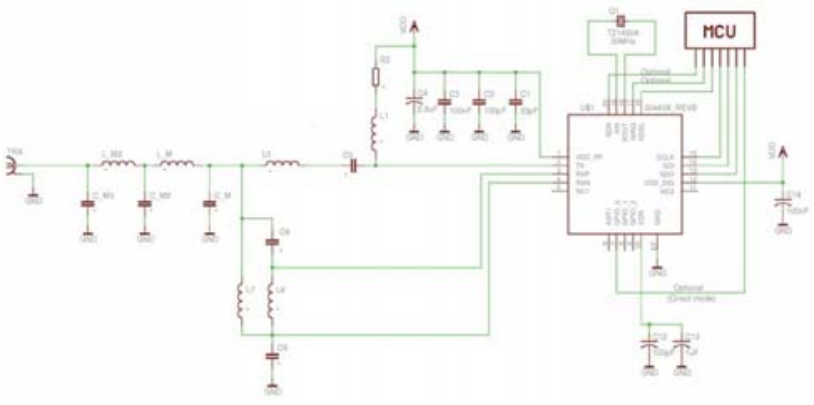

FIGURE II. CIRCUIT STRUCTURE DIAGRAM

RF chip SI4432 has multi-function, it is built-in frequency 
synthesizer, power amplifier, and low noise amplifier and phase locking modulator function modules. It is worth mentioning that transmission power and communication channel can be configured by the program. When chip in the receive mode, the peak current is not more than $13.7 \mathrm{~mA}$. The maximum transmitted power can be up to $+20 \mathrm{DBM}$, power consumption is lower than $75 \mathrm{~mA}$, and standby power consumption is low to $40 \mathrm{nA}$.

a) Working model: SI4432 working mode including standby mode, launch mode, receive mode, these patterns can be chosen or be set by the configuration register.

Launch mode: The data is sent through the SPI interface from MCU RF chips into the FIFO stack, implement the data of low speed into high speed launch process, is helpful to control power consumption

Device configuration can be showed by Silicon Labs' official register form.

Circuit layout: The PCB layout of Radio frequency part need to guarantee reliable signal transmission and reduce the internal and external electromagnetic interference. All the components will be put on the top layout in normal, and all the spare place will be covered with copper, connected the top layout and bottom layout with through holes, which is connected with the underlying ground.

In order to improve the EMC performance, the bottom layout's underlying ground can be designed reflux land, and the through hole will be set to minimum path points.

Thinking of power integrity, DC power supply and filter capacitor will put close to the VDD pin of RF chip. RF chip should have relatively independent power supply, which is advantageous to the stability of electricity supply, and also can strengthen the entire layout.

2) The part of temperature measurement: Temperature measurement circuit constitute with 3-wire PT100 positive temperature coefficient thermistor and $24 \Sigma-\Delta$ ADS1246 ADC chip made by TI Company (Figure 3). PT100 temperature range is $-200{ }^{\circ} \mathrm{C} \sim 850{ }^{\circ} \mathrm{C}$, and the RT expression is just like:

$$
\begin{gathered}
-200<\mathrm{t}<0^{\circ} \mathrm{C} \mathrm{RT}=\mathrm{R} 0[1+\mathrm{At}+\mathrm{Bt} \times \mathrm{t}+\mathrm{C}(\mathrm{t}-100) \mathrm{t} \times \mathrm{t} \times \mathrm{t}] \\
0 \leq \mathrm{t}<850^{\circ} \mathrm{C} \mathrm{RT}=\mathrm{R} 0(1+\mathrm{At}+\mathrm{Bt} \times \mathrm{t})
\end{gathered}
$$

$$
[\mathrm{A}=3.9083 \mathrm{E}-3, \mathrm{~B}=-5.775 \mathrm{E}-7, \mathrm{C}=-4.183 \mathrm{E}-12]
$$

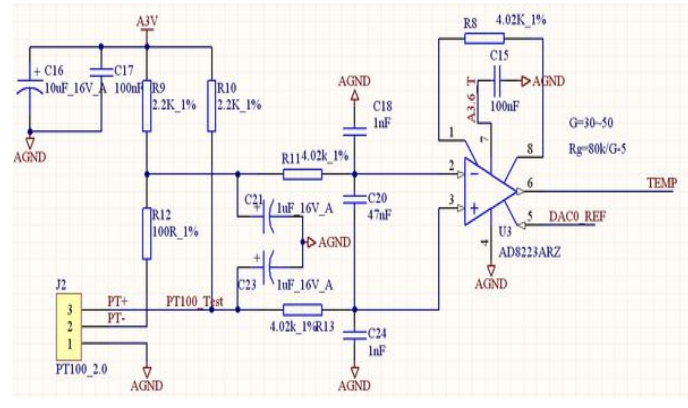

FIGURE III. CIRCUIT STRUCTURE DIAGRAM

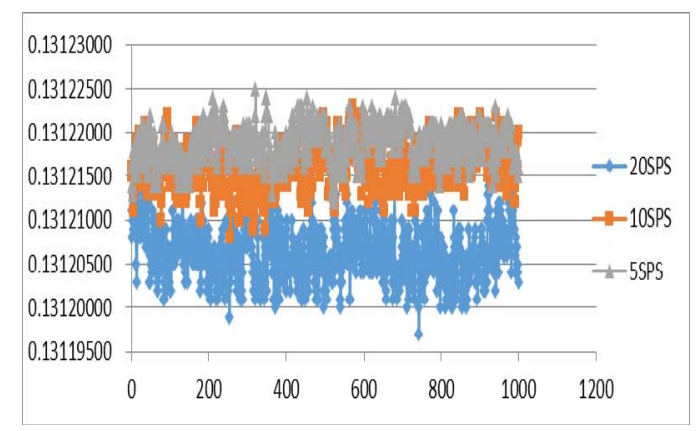

FIGURE IV. INTERNAL REFERENCE SOURCE

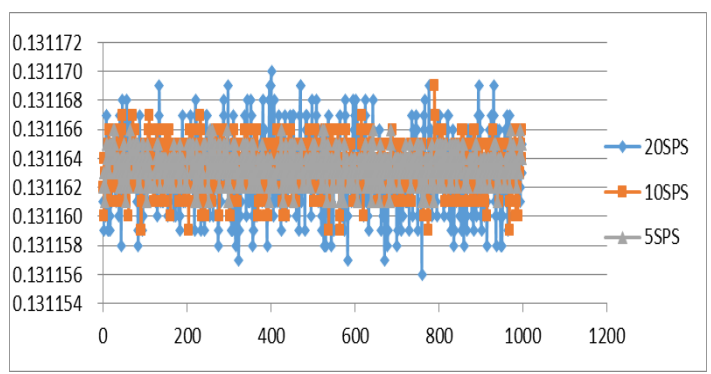

FIGURE V. INTERNAL REFERENCE SOURCE

It is not hard to see in conversion rate for 5 SPS, under the condition of using an external reference voltage source, ADS1246 can get effective resolution up to 19 , which can satisfy the required resolution $0.01{ }^{\circ} \mathrm{C}$ 16-bit $\mathrm{ADC}$ resolution requirement of design index.

a) Sleep mode: Through changing the level of the pin of START can convert mode of ADS1246. When sleep mode is available, the entire $\mathrm{AD}$ module power is not more than 0.4 uA.

b) MCU control circuit: This design USES batteries, has a strict requirements on the machine power consumption. Select TI Company's MSP430F149 ultra-low power MCU, which works in LPM3 sleep mode normally, timing waked up.

\section{B. Software Subsystem}

The software process is shown in Figure 5. Users can get target terminal real-time temperature information or back history information by intelligent router indirect. If you want to view the history of a particular section of the temperature information, you can issue the section through the enquiry instructions. If you want to set temperature time basis points, you can simply issue instructions of current accurate time, system will reset the target terminal of the current time. When the user issued successful, the target terminal will reply success and tag, and a preset rules will be set as an integral point. After the completion of the acquisition, system will set the latest temperature information data into the history of the cache queue first.

\section{Initializing:}

System self-checking $->$ MCU interface configuration-> ADS1246 Register configuration- $>$ SI4432 Register configuration(Receiving mode)- $>$ Register dormancy- $>$ MCU RTC start timing awaken and enter LP3 mode- $>$ system enter 
low power consumption mode

\section{Working:}

Time out $(0.5 \mathrm{~S})->\mathrm{MCU}$ into AM mode, detecting power, ->if power is low, alert; Else SI4432 into receiving mode->SI4432 will judge the received data just like set up sampling interval or real-time temperature query or a period of time temperature data query etc. and perform related instructions.

->sending the result->enter sleep mode (low power consumption ) ->timing. (Loop)

\section{PROCESS LOGIC}

1) The sensors' default rules are as follows:

a) Terminal automatically wake up and collect temperature data according to the pre-set time intervals;

b) Terminal automatically wake up every $0.5 \mathrm{~S}$, enter receiving mode, checking the wake-up frame come from router, if none in $10 \mathrm{~ms}$, system enter sleep mode, if received, continue to receive information frame, and if terminal does not received information frame in one second, system enter sleep mode. If received, system works according to the instruction from information frame. When finished instruction job, waiting $10 \mathrm{~ms}$ for new instruction, if there has no new instruction, system also enter sleep mode for saving power. Information frame instruction format as Figure 6. It including a set time instructions, set up automatic acquisition interval, real-time temperature query instructions and section temperature query. The end of each frame contains the LENGTH of DATA information, CMD, DATA segment CRC check results. The formula is just like:

$$
X^{16}+X^{12}+X^{6}+1
$$

\begin{tabular}{|c|c|c|c|c|c|c|}
\hline SYNC & STX & LENGTH & CMD & DATA & CRC & ETX \\
\hline 1 byte & 1 byte & 1 byte & 1 byte & 0.250 bytes & 2 bytes & 1 byte \\
\hline $\begin{array}{l}\text { Sync. } \\
\text { character }\end{array}$ & $\begin{array}{c}\text { Start of } \\
\text { transmission } \\
\text { character }\end{array}$ & $\begin{array}{l}\text { Frame } \\
\text { length }\end{array}$ & Command & Data & $\begin{array}{c}\text { Control } \\
\text { Redundancy } \\
\text { Check } \\
\text { LSB First }\end{array}$ & $\begin{array}{l}\text { End of } \\
\text { transmission } \\
\text { character }\end{array}$ \\
\hline OxFF & $0 \times 02$ & & & & & $0 \times 03$ \\
\hline
\end{tabular}

FIGURE VI. FRAME FORMAT

\section{CONCLUSION}

The designed wireless temperature sensor, in this paper, can be applied to various high precision temperature measuring occasions. System incorporates advanced wireless communication technology and low power technology, and can be extended to a complete network system through private ad-hoc network technology, it can be used not only in temperature collecting system, can also be extended to other measurement and control system. Through high-efficient power management, system greatly extend the continuous use time of the equipment, can be used in warehouse management and other fields.

\section{REFERENCES}

[1] Wang Dong, "Design and Application of Wireless Sensor Network; dissertation of Chongqing university", 2006-06.

[2] Li Cui Ran, Li Cheng Shu, "EAR protocol analysis of self-organizing wireless network", Guangdong communication technology, 2002, 22(3).

[3] Zheng Ceng Wei, Wu Chao Hui," Comparative Research of Several routing protocols in Wireless Sensor Network", Computer engineering and design, 2003,24(9):28-31.

[4] Sun Xue Bing, Zhou Zheng,"Research on target location algorithm in Wireless Sensor Networks", Computer engineering and Applications, 2004, 3:132-134

[5] ADS1246 DATASHEET (rev1.0).

[6] Wang Chao, Tang Hao, Huang Lin,'Temperature measurement and control system based on Pt100 type platinum resistance thermometers", Instrument technology, 2013,2:28-34.

[7] SI4432 Datasheet (rev1.0).

[8] MSP430X1XX Family User's Guide (2006)。

[9] Esrin D, Govindan R, Heidemann J., "Next century challenges: scalable coordination in sensor network [A]", Proceedings of the 5th Annual International Conference on Mobile Computing and Networks (MobiCOM'99) [C].Washington, USA.1999:263-270

[10] Zhang Sirong, Wang Yanping., "The modern cigarette factory construction innovation and practice" ,Beijing, China building industry press 